\section{The importance of particle}

\section{accelerators}

Ugo Amaldi, University of Milano Bicocca and TERA Foundation, Italy

$T$ o discuss the relevance of particle accelerators to society it is useful to distinguish the three main uses of their primary, secondary and tertiary beams:

1. analyses of physical, chemical and biological samples,

2. modifications of the physical, chemical and biological properties of matter, 3. research in basic subatomic physics. In this last application particles move close to the velocity of light and one should speak of 'massificators' rather than of 'accelerators'.

\section{Sample analyses and modification processes}

As far as the first two points are concerned, to a nice figure by $\mathrm{K}$. Bethge [1] I have added the leaves representing the many applications that continue to sprout from its various branches (Fig. 1).

The trunk has its roots in the nuclear physics of the thirties and the pull towards higher energies and beam currents still comes today from the study of fundamental particles and their interactions. Some of the accelerator technologies mentioned in the figure became mature so long ago that they are no longer discussed at large international conferences.

Already a cursory look at the figure proves that particle accelerators are very important, because they give irreplaceable contributions to many aspects of human life. The list of the fields of applications goes from $A$ to $Z$, i.e. from art to zoology.

In the field of the arts one can consider the work done at the Accélérateur Grand Louvre pour l'Analyse Élémentaire (AGLAE) on a drawing made around 1450 and attributed to Pisanello. By means of the PIXE technique the attribution was falsified, since securely attributable drawings are characterised by much less copper than the amount observed [2].

For the letter B we consider biology. After the completion of the decoding of the human genome, the attention of molecu-

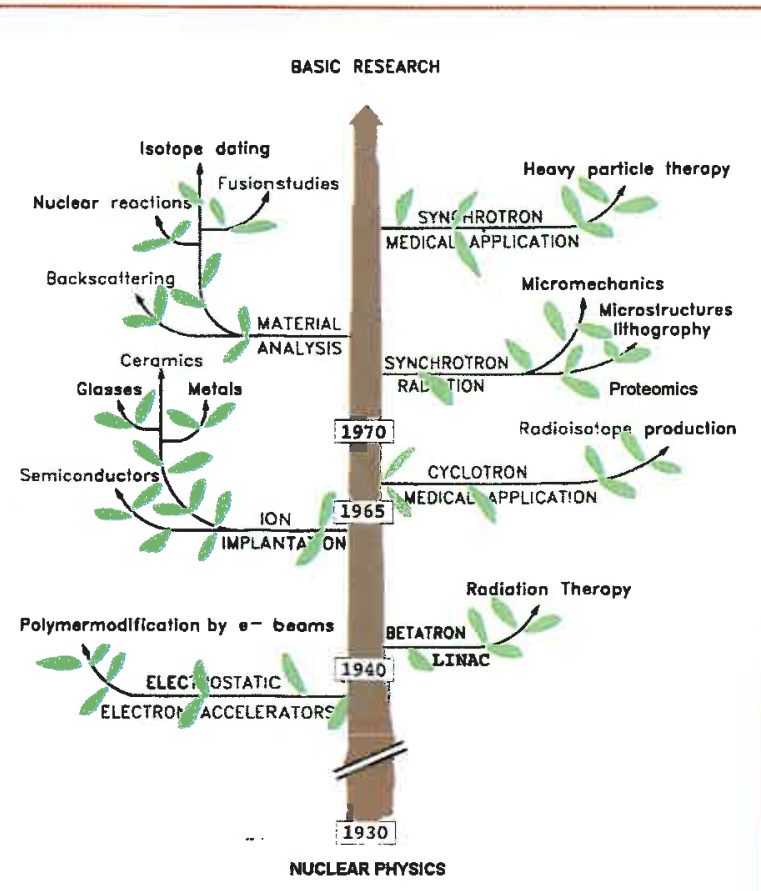

Fig 1: The Time Tree gives a pictorial view of the development of accelerators applications in both modification processes and sample analyses. sent the main tool in 'proteomics' and all suitable sources - in particular synchrotron radiation sources - are contributing and will continue to contribute.

Jumping for brevity down along the alphabet to medical diagnostics, cyclotrons are used to produce the isotopes used for Positron Emission Tomography (PET) and Single Photon Emission Computed Tomography (SPECT). Still, in about $80 \%$ of all examinations, isotopes (in par- are used. It is high time to use for this type of production $100-150 \mathrm{MeV}$ high-power cyclotrons instead of ageing reactors.

For curing some type of cancers, beams of $200 \mathrm{MeV}$ protons are used with established medical protocols since almost $30^{\prime} 000$ patients have been treated all over the world. At present the most promising new tool in radiotherapy is that form of hadrontherapy that utilises beams of light ions of atomic number around $Z=6$. At variance with protons, which interact with the cells essentially as X-rays, light ions better control the slowly growing radioresistant tumours which represents about $20 \%$ of all the tumours irradiated with Xrays. Since 1994 at HIMAC in Japan about 800 patients have been treated with carbon ions with satisfactory results; moreover in 1998 the carbon ion pilot project was completed at GSI, where about 50 patients have been irradiated. In the years 1996-1999 an AUSTRON-CERN-GSI-TERA Collaboration has carried out the Proton Ion Medical Machine Study (PIMMS), a study of a light ion synchrotron optimized for medical applications [4]. PIMMS is the basys of the therapy centres proposed by the by the TERA Foundation [5] and by the MedAUSTRON project [6] to their national authorities. Using PIMMS, preliminary proposals have also been prepared by TERA for the University Claude Bernard in Lyon and for the Karolinska Institute in Stockholm. Independently a hospitalbased therapy centre has been designed by GSI for the Heidelberg University [7].

In planetology small beams of low energy protons are used. At the Heidelberg Proton Microprobe the PIXE elemental mapping of micrometeorites is done with a spatial resolution of a few microns [8]. Coming finally to the letter $\mathrm{Z}$, in the field ticular Technetium 99m) produced at old reactors

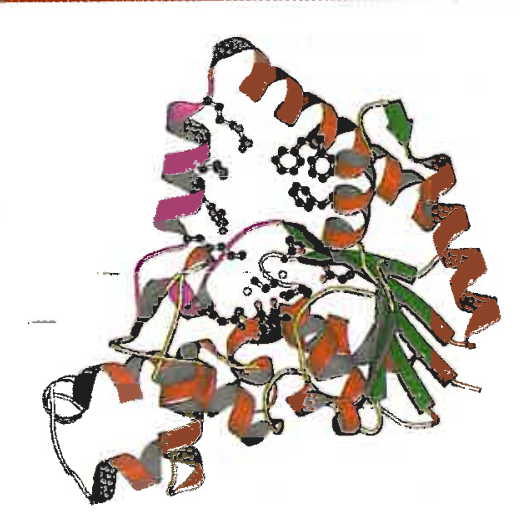

Fig 2: In 1996 the protein 5-exonuclease extracted from bacteriphage T5 was reconstructed from $X$-ray diffraction data collected at the ESRF [3]. 
of zoology one can quote the diffraction studies made at the European Synchrotron Radiation Facility of the isolated muscle fibre of a frog, excited at a frequency of 25 $\mathrm{Hz}$ [9].

The past spin-offs are impressive; what about the future? Spallation sources are moving to the unexplored territory of high power beams on target. Europe has a very good record with ISIS at RAL and two projects on the drawing table: the $E u$ ropean Spallation Source [10] and the AUSTRON project [11]. In the USA the new generation facility SNS will produce beams by the year 2005 [12].

A completely new application of great importance is the development of X-ray emitting Free Electron Lasers. A project is underway in the USA [13] and another one is a parallel development of the work done for the TESLA linear collider centred at DESY [14]. A $5 \mathrm{~Hz}$ electron beam of 15$50 \mathrm{MeV}$ formed of intense $(1 \mathrm{nC})$ and short ( $80 \mathrm{fs}$ ) bunches radiates photons through Self Amplified Spontaneous Emission when moving through a long undulator. At $1 \AA$ the peak brilliance will be at least ten orders of magnitude larger than the one achievable today.

Another recent developments centres on Rubbia's energy amplifier, a sub-critical lead-cooled fast fission reactor injected with the spallation neutrons produced by a powerful beam of $1 \mathrm{GeV}$ protons. The main use would be the incineration of radioactive wastes, but through the Adiabatic Resonance Crossing method [15] it could replace the ageing fission reactors in the production of Technetium $99 \mathrm{~m}$.

Finally one should not forget that the results of the experiments going on in Japan, the USA and France on inertial fusion induced by laser beams converging on a pellet cannot be directly applied to reactor construction due to the low laser efficiency. The only viable solution is based on short and powerful ion bunches. The accelerator systems required are complicated but the components are based on reasonable extrapolations of existing technologies [16].

All these examples show the importance of particle accelerators in art, medicine, industry and nonnuclear sciences. The argument becomes even stronger when the number of accelerators is considered.

Table 1 shows that $55 \%$ of the $15^{\prime} 000$ accelerators running at present in the world are devoted to modification processes: ion im-

plantation, surface modification and industrial applications (mainly sterilisation and polymerisation). Electron linacs used in radiotherapy represent one third of all the existing accelerators. There are about 70 synchrotron radiation sources in the world, with the highest density in Japan, and more than 100 particle accelerators used for research in subatomic physics. In 1994 the total number of accelerators was about 10000 , so that one can conclude that the progression rate is about $15 \%$ per year.

Status of subatomic physics and its future accelerators

Subatomic physics covers two subjects: nuclear physics and particle physics. The former is concerned with systems having baryon numbers much larger than 1 and naturally subdivides in two chapters. The content of the first chapter, titled high-temperature nuclear physics, is best discussed with reference to the phase diagram of Fig. 3.

On the horizontal axis the relative baryon densities of the smaller (the atomic nuclei) and the largest nuclear systems (the neutron stars) are indicated. On the vertical axis the temperature of systems having baryon number definetely larger than 1 is plotted in terms of the component kinetic energy, the correspondence being 100 $\mathrm{MeV}=10^{12} \mathrm{kelvin}$. The problem here is the experimental study of the deconfinement transition predicted by the Standard Model description of the baryons in terms of quarks bound by the continous exchange of gluons. The oblique arrows indicate the approximate paths of the non-equilibrium baryonic systems studied by colliding

\begin{tabular}{|l|c|}
\hline CATEGORY & NUMBER \\
\hline Ion implanters and surface modifications & $7^{\prime} 000$ \\
\hline Accelerators in industry & $1^{\prime} 500$ \\
\hline Accelerators in non-nuclear research & $1^{\prime} 000$ \\
\hline Radiotherapy & $5^{\prime} 000$ \\
\hline Medical isotopes production & 200 \\
\hline Hadrontherapy & 20 \\
\hline Synchrotron radiation sources & 70 \\
\hline Research in nuclear and particle physics & 110 \\
\hline \multicolumn{2}{|c|}{ TOTAL } \\
\hline
\end{tabular}

Table 1: Accelerators in the world. The data have been collected by W. Scarf and W. Wiesczycka [17]. heavy ions with heavy fixed targets at the AGS (Brookhaven) and at the SPS (CERN).

Recently the physicists working at the SPS have put together all the information, collected by many different experiments in ten years of work, coming to the conclusion that a new state of matter has been observed. The results soon expected from RHIC should bring very clear information on the deconfinement transition starting from temperatures which are about two times larger than the transition temperature $(200 \mathrm{MeV})$. The lead-lead collisions to be studied with the ALICE experiment at the LHC will start with much larger temperatures, thus reproducing even more closely what happened in the early Universe. From this point of view it can be said that, if no other higher temperature transition is discovered, there will be no need of a ion-ion collider of even greater energy.

Fig. 4 reproduces the chart useful to describe the main issues encountered in the second chapter, which can be called 'low-temperature nuclear physics'.

The dark and shaded area cover the well-studied stable and unstable 


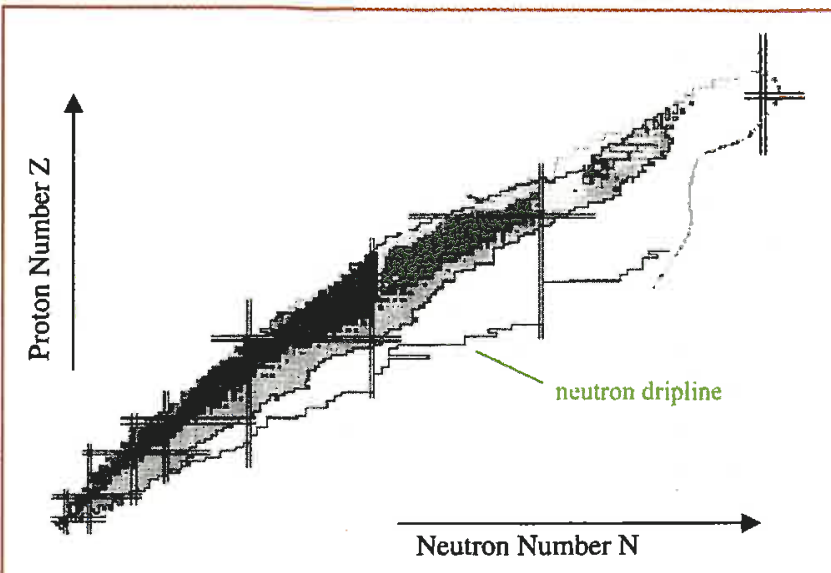

Fig 4: In this standard chart the nuclei that are below the neutron dripline are not bound. The crosses indicate the magic nuclei.

nuclei. Below, bounded by the neutron dripline one sees the area of neutron-rich nuclei that can be produced only by accelerating and colliding unstable (radioactive) nuclei with fixed targets. The frontier instruments of low-temperature nuclear physics are thus accelerators of radioactive nuclei. The phase space to be explored is very large, as indicated by the extension of the unknown white area lying above the neutron dripline.

The importance of these experiments also comes from the fact that here the socalled ' $r$-process' takes place, i.e. the rapid neutron capture happening in supernovae and leading to the creation of many elements heavier than iron.

The links between subatomic physics and astrophysics are even stronger. Indeed all that one learns on the second subject of subatomic physics - i.e. 'particle physics' is crucial for solving the first of the three really important scientific problems: the origin of the Universe, the origin of life and the origin of consciousness. This is possible because simple thermodynamic considerations connect the universal time $t$ with the energy $E$ available in the collisions of the particles forming the primordial soup:

$$
t_{\text {microseconds }} \cong 1 / E^{2} \mathrm{GeV} .
$$

The collision and decay phenomena happening both at the beginning of the Universe and now around our massificators are determined by elementary emission and absorption processes that are governed by the numerical value of $\alpha^{-1}$, the inverse of the coupling.

The reason is that for every interaction (strong, electromagnetic and weak) $\alpha^{-1}$ equals the number of times one has to observe the matter-particle before finding a virtual mediator close to it. If $\alpha^{-1}$ is of the order of 1 , the matter-particle is always surrounded by a mediator and the force is strong.

The main point is that the value of $\alpha^{-1}$ depends upon the energy (or the momentum) $E$ of the virtual force-particle. Indeed, when a blob of energy-momentum $\mathrm{E}$ surrounds a matter-particle, all particle-antiparticle pairs of energy smaller than $E$ can be created for a very short time. They influence the interaction between the mediator and the matter-particle and change the value of $\alpha^{-1}$. For instance, when a virtual photon of momentum larger than about $10 \mathrm{GeV}$ is exchanged between two electrons passing by, the couplings are modified by the momentary creation of beauty-antibeauty quarks, each having a mass of about $5 \mathrm{GeV}$.

Quantum field theory allows precise calculations of the energy dependence of all couplings, once their values at a given energy are known together with the masses of all the particles that can temporarily be created. In 1991 the new accurate LEP measurements of the three fundamental couplings performed at $\mathrm{E} \cong 100 \mathrm{GeV}$ allowed for the first time their precise extrapolation [18] and a fact suspected for a few years became a certainty: in the Standard Model the forces do not unify at high energy. But unification is obtained if a theoretically more satisfactory model is adopted, the Supersymmetric (SUSY) Mod-

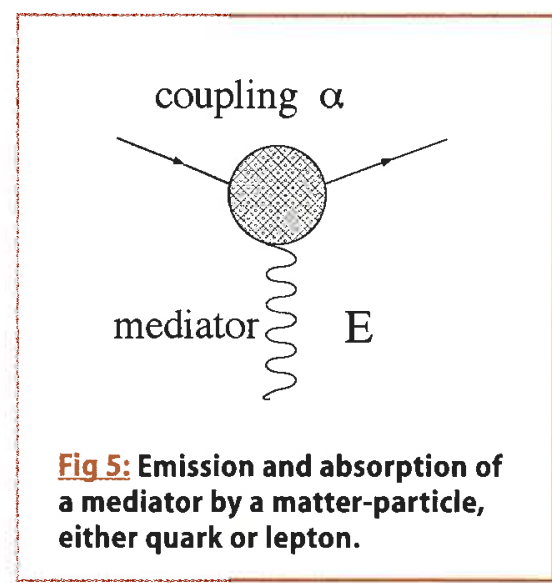

\section{Call for papers}

"Europhysics Letters is still fairly young and our com-

EUROPHYSICS LETTERS munity changes its publishing habits rather reluctantly. However, I think a European publication belonging to all of us represents a way for everyone to invest in the future. So there is no other direction but ahead, and with more of us being involved. The first ten years have been a very good omen for predicting the success of this precious investment." F.A. Gianturco, Editor in Chief, Europhysics News, 6 (1996) 233

Through a real European collaboration, Europhysics Letters is one of the top ten physics journals in terms of impact factor world wide (source: ISI, 1999). The Journal receives contribution from all over the world, from more than 40 countries, and is circulated in about thousand examples to more than 30 countries. The articles and activity of EPL are of direct benefit to all members of the European Physical Society.

So why not submit your Letters to EPL?

Letters submitted to EPL should contain non-trivial new results, ideas, concepts, experimental methods, theoretical treatments etc. and be of broad interest and importance to one or several sections of the physics community. The presentation should satisfy the specialist, yet remaining understandable to the researchers in other fields through suitable, clearly written introduction and conclusion. Your Letter should not have been submitted simultaneously to another journal. There are no page-charges and authors will receive $\mathbf{5 0}$ reprints gratis.

The manuscript should contain a title page, an abstract, main text, acknowledgements, references followed by captions of eventual figures and tables. The length of the manuscript should not exceed 7 journal pages. When preparing your manuscript, author guidelines are available, as well as macros for formulas developed in EUROTeX.

Papers may be submitted electronically, or in paper form by mail (the original and three copies).

For more information on how to submit your Letters, please see the EPL web site at www.epletters.ch, or contact Mrs. E. Thomas, Staff Editor, Europhysics Letters, chemin de la Vendée 27, P.O. Box 69, CH - 1213 Petit-Lancy 2,email edith.thomas@epletters.ch 


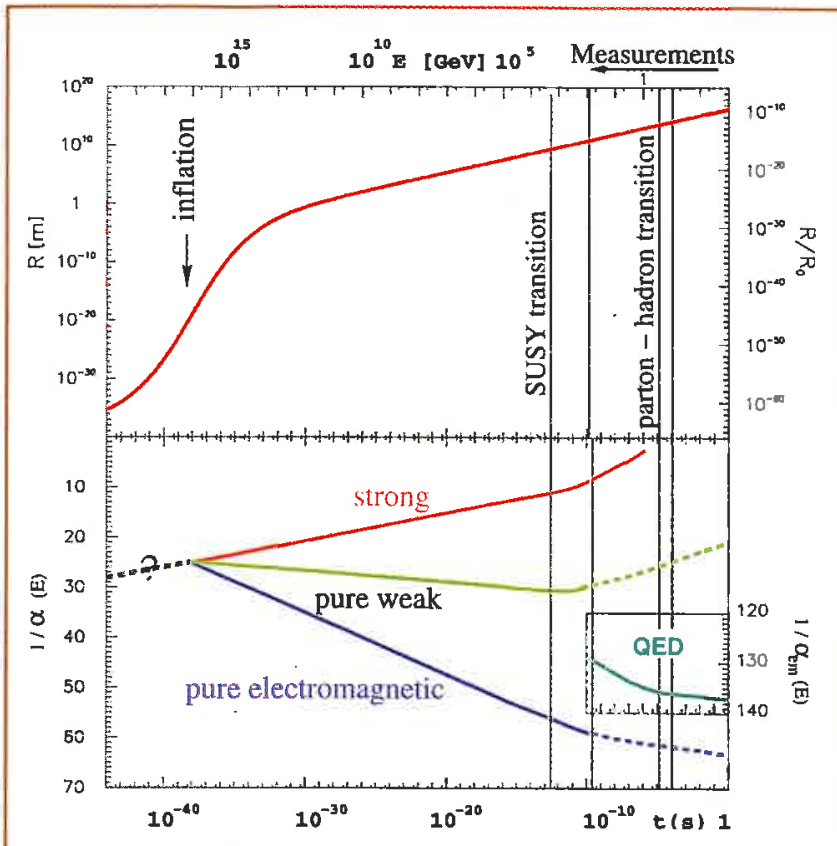

Fig 6: The lower graph shows the possible times of occurrence of the SUSY transition (i.e the disappearance of the superparticles) and of the partonnucleon transition (i.e. the disappearance of the quarkgluon plasma). As indicated by the upper scale, in this graph energy goes from right to left, so that the universal time goes from left to right as indicated on the lower scale. The upper panel represents the inflation of the early Universe, which probably happened at the time of the divergence of the three fundamental couplings.

el in which there is full symmetry between the matter-particles (quark and leptons) and the force-particles (the boson transmitting the fundamental forces). In the minimal version of such a model - as in other less simple ones - new 'superparticles' are predicted at energies just above the about $100 \mathrm{GeV}$ explored till now. Their existence would modify the energy dependence of the couplings, bending them towards a unification point at $10^{16} \mathrm{GeV}$, as shown in the lower part of Fig. 6. (Note that in this graph the energy scale goes from right to left and that experiments have been done only in the energy region marked 'measurements'.)

Fig. 6 shows that in this model the couplings diverged, at $10^{-38} \mathrm{~s}$, and at $10^{-12}-10^{-10} \mathrm{~s}$ a transition took place, during which all superparticles decayed and could not be created any longer for the lack of energy in the collisions. (It is however possible that the lightest neutral superparticles did not decay and form today the so-called 'dark matter'.) Also other models of the events before $10^{-10} \mathrm{~s}$ are fashionable today. Anyway we know that around that time all the $\mathrm{W}$ and $\mathrm{Z}$ weak bosons decayed. Since then the pure weak and pure electromagnetic forces are mixed together to form the usual weak and electromagnetic forces (inset of Fig. 6). The only way to probe the possible SUSY transition is to study collisions at energies larger than $100-200 \mathrm{GeV}$. This is the energy domain of the LHC.

The matterparticles of the Standard Model are the second hot issue in particle physics. For the quarks the phenomenology is quite well known. In the collisions processes, the down, strange and beauty quarks are produced, but in the decay processes linear combinations of them - indicated as $d^{\prime}, s^{\prime}$ and $b^{\prime}$ - give the observed effects. The CabibboKobayashi-Maskawa matrix connects them and it took more than forty tears to have measurements of reasonable precision (Fig. 7). The three neutrinos are expected to display a similar behaviour. This is, in a sense, a theoretically 'simpler' and thus even more interesting phenomenon to constrain the hopefully 'final' theory that will embrace and supersede the Standard Model, since neutrinos do not feel strong interactions.

Quark mixing can be studied at colliders because all heavier quarks decay within a few millimetres. Neutrinos, being the lightest of all particles, do not have enough energy to decay and thus, once produced, travel for ever and one has to perform 'oscillation experiments' with very intense neutrino beams and long flight-paths. We already know the order of magnitude of some non-diagonal elements of the neutrino matrix, but only much better measurements will allow to falsify any theory of everything': certainly more than fifty years of experimental work will be needed. These are the arguments that in the last years have prompted the design of neutrino factories. They will become widely required accelerator complexes to be built together with the more standard hadronic and leptonic colliders in our accelerator centres, eventually sending beams to far away underground laboratories in a possible World Wide Neutrino Web.

This field is in full development while the future of lepton and hadron colliders is well defined. Precise experiments on the quark sector will continue at quark factories and possibly at a new Z-factory. As far as the high-energy frontier is concerned, while LHC experiments will be running, at least one high-energy electron-positron linear collider will have to be built to study in detail the new energy domain explored with the wide-band but less precise proton-proton processes. The energy attainable should be as large as feasible; the results obtained at LEP, Tevatron and LHC will indicate the optimal initial energy. Muon colliders are for later, when neutrino factories will have opened the way to intense muon sources.

It is interesting to remark that targets absorbing many megawatts of power carried by high energy proton beams are needed both for fundamental research (neutrino and muon factories), for research in non-nuclear sciences (spallation sources) and for energy and medical applications (accelerator driven subcritical reactors). This is a wonderful example of unexpected synergetic developments.

$$
\begin{aligned}
& \left(\begin{array}{l}
d^{\prime} \\
s^{\prime} \\
b^{\prime}
\end{array}\right)=\left(\begin{array}{ccc}
0.9740 \pm 0.0010 & 0.2196 \pm 0.0023 & \mathbf{0 . 0 0 4 0}+0.0006 \\
0.224 \pm 0.016 & \mathbf{0 . 9 1} \pm \mathbf{0 . 1 6} & 0.0402 \pm \mathbf{0 . 0 0 1 9} \\
<0.010 & \simeq 0.0400 & 0.99 \pm 0.29
\end{array}\right) \\
& \left(\begin{array}{c}
\nu_{e}^{+0.0006} \\
\nu_{\mu} \\
\nu_{\tau}
\end{array}\right)=\left(\begin{array}{ccc}
-c_{12} c_{13} & c_{13} s_{12} & s_{13} \\
-c_{23} s_{12} e^{i \delta}-c_{12} s_{13} s_{23} & c_{12} c_{23} e^{i \delta}-s_{12} s_{13} s_{23} & c_{13} s_{23} \\
s_{23} s_{12} e^{i \delta}-c_{12} c_{23} s_{13} & -c_{12} s_{23} e^{i \delta}-c_{23} s_{12} s_{13} & c_{13} c_{23}
\end{array}\right)
\end{aligned}
$$

Fig 7: The experimental CKM matrix connecting the quarks is given by neglecting the $C P$ violating phase. A parametrisation of the neutrino mixing matrix is also shown. 


\section{Conclusion}

Particle accelerators are important for the arts, the other sciences, medicine and high-tech industries so that their overall number increases by about $15 \%$ per year. The high energy ones are crucial for the understanding of the origin of our Universe and of the formation of heavy nuclei, without which the Earth could not exist. Moreover they give the information needed to falsify any new 'theory of everything'. Thus, the trunk of the Time Tree is strong and developing, the old leaves are green and new leaves are sprouting. If the accelerator specialists continue to invent and labour as in the past, the future of particle accelerators and massificators is secured.

\section{References and notes}

[1] K. Betghe, Nucl. Phys. News, 9/1 (1999) 20.

[2] A. Duval, in Pisanello, Actes du Colloque du Juin 1996, D. Cordellier and B. Py eds., Musèe du Louvre, La Documentation Française, 1998.

[3] T.A. Ceska, J.R. Sayers, G. Stier, D. Suck, Nature 382 (1996) 90.

[4] P.J. Bryant et al, Proton-Ion Medical Machine Study, Part I and II, CERN/PS 1999-010 (DI) and CERN/PS 2000-007 (DR). A CD is available.

[5] The National Centre for Oncological Hadrontherapy at Mirasole, U.Amaldi ed., INFN, Frascati, 1997.

[6] Med-AUSTRON Machbarkeitsstudie, R. Pötter, T. Auberger and M. Regler eds., AUSTRON, Wiener Neustadt, 1998.

[7] Proposal for a Dedicated Ion Beam Facility for Cancer Therapy, K.D. Gross and M. Pavlovic eds., Univ. HeidelbergDKFZ-GSI, GSI, Darmstadt, 1998.

[8] P. Arndt et al, J. Conf. Abs., Cambridge, 1, (1996) 24 .

[9] I. Dobbie et al, J. Muscle Res. Cell Motility, 17 (1996) 163.

[10] www.fz-juelich.de/ess/

[11] www.ati.ac.at/austron/

[12] www.sns.gov

[13] www-ssrl.slac.stanford.edu/lcls/

[14] www.desy.de/ wroblewt/scifel/

[15] H. Arnould et al, Phys. Lett. B458 (1999) 167.

[16] www.gsi.de/gsi.research.html

[17] Data by W. Scarf and W. Wiesczycka reproduced in U. Amaldi, Proc of EPAC 2000 , in print.

[18] U. Amaldi, H. Fürstenau and W. de Boer, Phys. Lett. B260 (1991) 447. 